\title{
Morphology Directionality
}

National Cancer Institute

\section{Source}

National Cancer Institute. Morphology Directionality. NCI Thesaurus. Code C117622.

A qualifier for the direction on the body the morphology assessment is performed. 